\title{
Non-invasive Measurement of Biomechanical Properties of in vivo Soft Tissues
}

\author{
Lianghao Han, Michael Burcher, and J. Alison Noble* \\ Medical Vision Lab, Department of Engineering Science, Oxford University \\ \{lhhan, burcher, noble\} @robots.ox.ac.uk
}

\begin{abstract}
Quantitative descriptions of in vivo biomechanical properties of soft tissues are necessary for tissue evaluation and a meaningful surgical simulation. A hand-held ultrasound indentation system that can acquire force-displacement response in vivo has been developed. Using this system, non-invasive measurements of in vivo biomechanical properties of tissues are described in this paper. First, a linear elastic model was used to describe a porcine phantom material. Its Young's modulus was estimated via a mathematical solution from force-displacement curves. The estimated value of Young's modulus was in good comparison with those from a material test machine and 2D and 3D finite element simulations. Secondly, a finite element-based inverse scheme was used to reconstruct Young's modulus distribution of a three-layer phantom based on the displacement field measured from 2D continuous ultrasound images. Finally, in our primary study a pseudo-elasticity model was used to fit the experimental data of in vivo breast tissue.
\end{abstract}

\section{Introduction}

Soft tissue abnormalities are often correlated to a local change in mechanical properties. For instance, physicians use palpation widely as a qualitative diagnostic tool for breast cancer. However, this technique is subjective. More quantitative descriptions of in vivo biomechanical properties of soft tissues are needed for successful clinical applications in surgical simulation and planning, minimally invasive and tele-surgery, and image guided surgery and diagnosis. The biomechanical properties of soft tissues vary significantly from one individual to another, and can take on different values depending on whether they are measured in-vivo or in vitro. Unfortunately, most research has been focused on in vitro measurements of soft tissue under different loading conditions [1,2]. Very little quantitative information is available on the biomechanical properties of soft tissue in vivo in the literature $[3,4]$.

A common method of assessing biomechanical properties involves using an indentation test, a procedure where an indenter depresses the tissue and the resulting deformation of the external surface is recorded. Indentation tests have been widely used for measuring material properties of soft tissues [4,6,7]. Because tissue's thickness will affect the indentation response [8,9], it is necessary to measure it. Using a combination of ultrasound techniques and some theoretical models $[8,9]$, hand-held

\footnotetext{
* Correspondence to: Dr J.A. Noble
} 
ultrasound indentation devices $[5,10]$ have been proposed to measure in vivo biomechanical properties of soft tissue, in which ultrasound techniques were used to measure tissue's thickness. The above indentation devices with a single element transducer were restricted to one-dimensional testing, and limited in investigating internal components of tissues. Recently, some reconstruction approaches $[11,12,13,14]$ under the framework of inverse-problem solutions have been proposed to quantitatively reconstruct the material properties of tissues based on 2D internal deformation (displacement or strain) fields induced from an externally applied compression. In these methods, the displacement or strain field was obtained from a sequence of medical images (MRI or ultrasound). Combining indentation test devices, imaging devices and reconstruction algorithms, it is possible to investigate biomechanical properties of abnormal and normal components of in vivo tissues.

A 3D free-hand ultrasound imaging system with a force transducer was used for deformation correction in ultrasound imaging to produce an improved 3D reconstruction [15]. In the current study, this system was used as a novel hand-held ultrasound indentation system to measure in vivo material properties of tissues. Non-invasive measures of biomechanical properties on simulation material and in vivo breast tissue are described in this paper.

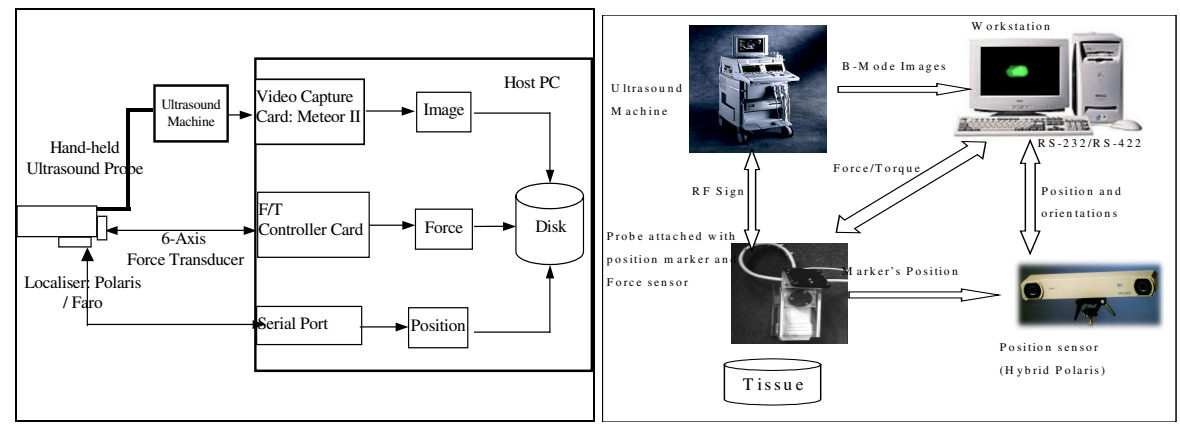

(a) System architecture

(b) Block diagram of indentation system

Fig. 1. Free-hand Ultrasound Indentation System

\section{Material and Methods}

\subsection{System Description}

A force transducer (Mini 40, ATI Industrial Automation) was mounted on a 3D freehand ultrasound system developed in our lab to form an ultrasound indentation test system in which the ultrasound probe attached with a force sensor and 4 infrared LED's (position marker) formed a hand-held indenter. Figure 1(a) shows the system architecture. Figure. 1(b) presents the schematic diagram of the ultrasound indentation system. The ultrasound (B-mode) images were obtained using a conventional ultrasound machine (Sonos 5500(Agilent Technologies)) and a 7.5MHz linear array probe (Hewlett Packard L7540). A meteor II-MC frame grabber (Matrox Imaging, Dorval, Canada ) was used to capture frames from the video output of the ultrasound machine. The position and orientation of the probe was recorded using a Polaris hybrid optical 
tracker (Northern Digital Inc, Ontario, Canada) that uses a stereo camera to measure the position of 4 infrared LED's mounted on the probe.

Software has been written in $\mathrm{C}++$ that interfaces with the hardware components and controls the acquisition. The code is multi-threaded with separate threads acquiring video, position and force data. This allows the recording to occur asynchronously at the maximum rates determined by the acquisition hardware: $25 \mathrm{~Hz}$ for video, $60 \mathrm{~Hz}$ for position and force measurements.

\subsection{Phantom}

Two types of block-shaped phantoms were made to simulate soft tissue. One contained one layer, the other one contains three layers. Each layer was constructed of gelatin powder from porcine skin (G-2500, Sigma Ltd.). The gelatin powder was hydrated with deionized water and heated above its gel point to disperse the collide, clarify the solution and release trapped gases. Then, the liquid gel solution was cooled and poured into a box mould kept in an ice-water bath. While the gel solution was still in liquid form, talcum powder (Johnson's Ltd.) was added to increase the absorption and scattering. The phantoms were then stored at $10^{\circ} \mathrm{C}$. The one-layer phantom was constructed from $5 \%$ by weight gelatin. The three-layer phantom was constructed from $6 \%, 2 \%$ and $4 \%$ by weight gelatin from top to bottom (Fig.2). The one-layer phantom was of dimensions $120 \mathrm{~mm}$ wide, $190 \mathrm{~mm}$ long and $42 \mathrm{~mm}$ high; the threelayer phantom was of dimensions $120 \mathrm{~mm}$ wide, $190 \mathrm{~mm}$ long and total $33.5 \mathrm{~mm}$ thick (first layer $8.7 \mathrm{~mm}$, second layer $10.8 \mathrm{~mm}$, third layer $14 \mathrm{~mm}$ ).

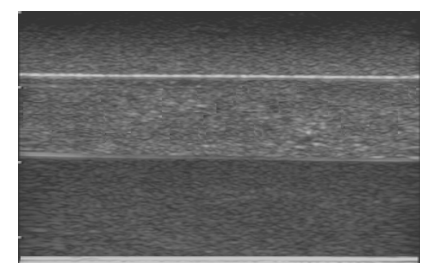

(a) Initial contact

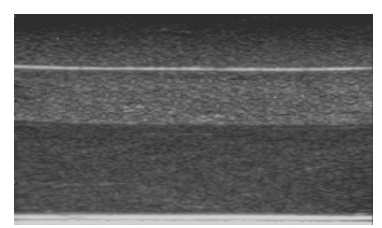

(b) Larger strain

Fig. 2. B-mode images of a three-layer phantom at different compression

\subsection{Measurement Procedure}

The US-based indentation system was applied to phantoms and the breast of a volunteer. First, the probe was placed on the surface of the specimen with a minimal force. Then, the load was gradually increased manually and then removed. This was repeated a few cycles in each trial. Ultrasonic coupling gel (Parker Laboratories, NJ, USA) was spread on the contact surface of each specimen prior to indentation. The phantom was compressed with indentation rates approximately, $1-2 \mathrm{~mm} / \mathrm{s}, 2-3 \mathrm{~mm} / \mathrm{s}$, and $5-10 \mathrm{~mm} / \mathrm{s}$ to investigate the effect of loading rate on measurement values. The maximum indentation depth was kept to within $20 \%$ of the initial thickness. During each experiment, the load, the probe position and 2D B-mode image (Fig.2) measurements were acquired simultaneously and stored to the computer disk. From the 
position information of the probe obtained with optical tracking system, via a coordinate transformation, the indentation depth was obtained. The tissue depth also could be measured from the B-mode images under the assumption that the speed of sound in soft tissue is a constant $\left(1540 \mathrm{~ms}^{-1}\right)$. In Fig. 2, B-mode images of one threelayer phantom are shown at different strains. Because there were unknown time delays in the position sensor, force sensor, ultrasound machine and frame grabber recordings, it was necessary to calibrate for the relative time delay between the storage of the ultrasound scan and the position measurement. In this paper, we employed the same method used by Prager et al [16].

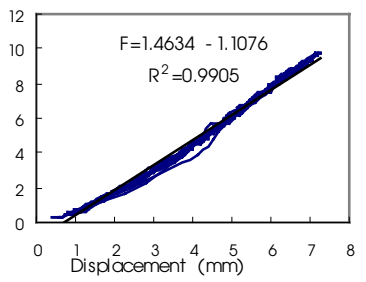

(a) Strain ratio $<1-2 \mathrm{~mm} / \mathrm{s}$

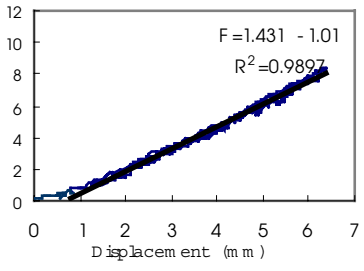

(b) Strain ratio $2-3 \mathrm{~mm} / \mathrm{s}$

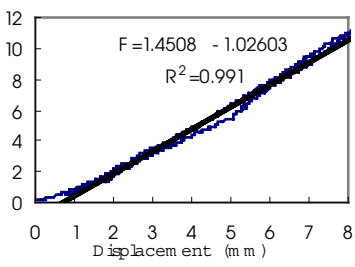

(c) Strain ratio $5-10 \mathrm{~mm} / \mathrm{s}$

Fig. 3. The force-displacement curves of a one-layer porcine phantom

\section{Results}

\subsection{Single-Layer Phantom}

Figure 3 shows experimental force-displacement curves of the one-layer phantom for three indentation rates. At the beginning of loading, the force-displacement curve shows slightly nonlinear behavior because of the incomplete contact between the probe and the phantom. When the indentation depth increases to about $1.0 \mathrm{~mm}$, the probe and the phantom contact each other completely. The response of phantom to loading is now linear and nearly independent of the indentation rate. This demonstrates that the phantoms behave as elastic materials i.e. the viscous component is negligible.

The Young's modulus $E$ can be calculated using a mathematical solution to the contact problems of a rectangular block on an elastic layer with finite thickness $[17,18]$.

$$
E=2\left(1-v^{2}\right)\left(\frac{2 a p}{\delta}\right) \kappa(v, h / a)=2\left(1-v^{2}\right)\left(\frac{F}{\delta}\right) l^{-1} \kappa(v, h / a)
$$

Here, $v$ is Poisson's ratio, $F$ the total applied force, $l$ the probe's length, $a$ the indenter half-width, $p=F / a l, \delta$ the indentation depth, $h$ the tissue thickness, and $\kappa$ a scaling factor. The scaling factor $\kappa$ provides a theoretical correction for the finite thickness of the elastic layer, and it depends on both the aspect ratio $h / a$ and Poisson's ratio $v$. Assuming phantoms to be nearly incompressible, the Poisson's ratio is taken to be 0.495 in the study, then $\kappa=\ln (2 \mathrm{~h} / \mathrm{a})-0.976+1.02(\mathrm{~h} / \mathrm{a})^{-2}-1.14(\mathrm{~h} / \mathrm{a})^{-4}$ [18]. The assumption of near incompressibility was made based on the fact that most tissue fluid would not have enough time to move within the tissue under a quick indentation 
[19]. The ratio $F / \delta$ is determined by the slope of the load-indentation response shown as Fig.3. The value of Young's modulus of one layer phantom was $28.1 \mathrm{KPa}$ from Eq.(1). The measured Young's modulus 28.6 KPa from a standard material test machine verified the above value.

The experimentally measured Young's modulus was also verified using two finite element(FE) models shown as Fig 4. Because the length $(50 \mathrm{~mm})$ of contact area between the US probe and the phantom surface is larger than its width $(14 \mathrm{~mm})$, a $2 \mathrm{D}$ FE model under the plane strain assumption was used to find the Young's modulus. A 3D FE model was also used. Four-node elements and eight-node elements (ABAQUS, HKS Inc. Pawtucket, RI) were created for 2D and 3D analysis, respectively. The interface between the US probe and the phantom was assumed to be frictionless because friction has a negligible effect on the indentation process for a thick-layer tissue[18]. Based on an elastic assumption, the Young's modulus of a homogeneous structure has a linear relationship with indentation depth under the same boundary condition. The Young's moduli determined by 2D and 3D FE analysis were 27.2 $\mathrm{KPa}$ and 29.8KPa,respectively.

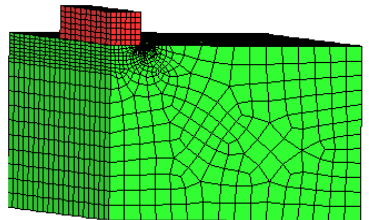

(a)3D Mesh

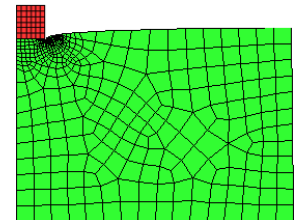

(b) 2D Mesh(Deformed)

Fig. 4. Finite element meshes

\subsection{Multi-layer Phantom}

To demonstrate how the same indentation probe can be used to estimate Young's moduli of different components of a tissue, a three-layer phantom as shown in Fig.2 was indented by the ultrasound probe. The internal deformation of three layers was measured by tracking the boundary lines between two different layers from a sequence of B-mode images [20]. The percentage deformation of the three layers is plotted against the total applied force in Fig. 5. Note that the second layer is softer than other two layers, and hence its percentage deformation is the largest. The first layer directly contacts with the probe, it is subject to a much larger local stress. Therefore, although the first layer is harder than the third layer, its percentage deformation is very close to that of the third layer. The Young's modulus of each layer in this phantom cannot be measured directly. To reconstruct the Young's modulus distribution, a finite element based inverse method has been developed [14]. An objective function relating the least-square difference of model-predicted and measured displacement fields from a sequence of 2D B-mode images is minimized with respect to the unknown Young's moduli. The solution can be obtained by iteratively adjusting the Young's moduli in the FE mode until the model-predicated displacements most closely match the measured displacements from ultrasound images. In order to obtain a physically meaningful solution, the Young's moduli are bounded with the lower and upper limits that are not considered in other research groups $[11,12,13]$. The finite element method acts as a forward problem solver to calculate the displacement field 
of a simplified 2D plane-strain model. A modified Levenberg-Marquardt method and an active set strategy are used to solve this constrained nonlinear optimization problem. For more details on constrained optimization, see [21]. Using this method the ratio of the Young's moduli among three layer materials was reconstructed as 1:0.298:0.562, which was comparable to 1:0.256:0.498 measured from three singlelayer phantoms corresponding to three layers of the phantom respectively.

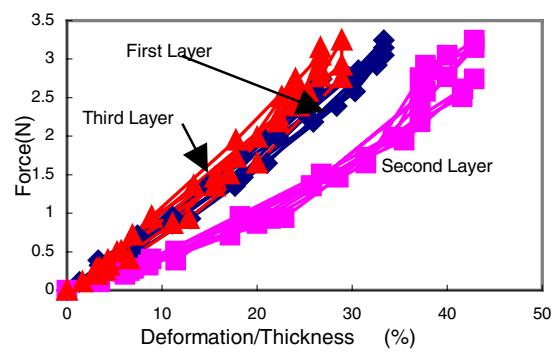

a. The percent deformations of each layer

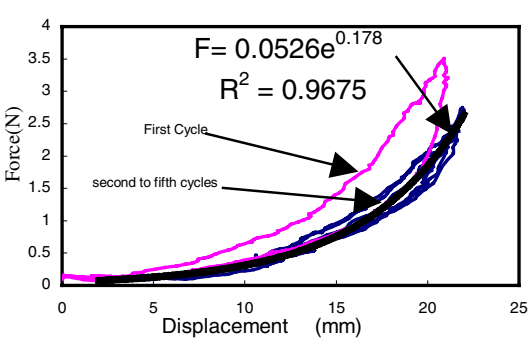

b. Typical loading-unloading curves of a volunteer's breast with five cycles

Fig. 5. The Force-deformation curves

\subsection{In vivo Test on Breast Tissue}

Figure 5(b) shows the relationship between the force and external cyclically varying strain under the compression of the probe on the breast of a volunteer. The effect of preconditioning [1] of in vivo breast tissue is evident. With an increased number of cycles of force, the hysteresis decreases while the curve shifts to right. After around 2 cycles a steady state is reached. The breast tissue in vivo exhibited visoelasticity, linear and elastic model was no longer suitable to describe this material. Because the differences between the loading and unloading curves(Fig. 5(b)) after the first cycle are not large, we can describe the properties of the soft tissue by a pseudo-elasticity model[1]. Force and deformation response can be represented as $F(\lambda)=\alpha e^{\beta \lambda}$, in which $\lambda$ is the stretch ratio $\delta / \delta_{0}, \delta$ and $\delta_{0}$ are the compression depth and initial thickness, respectively, and $F$ is the measured force. Taking the derivative $d F / d \lambda=\beta F$ results in parameter $\beta$ as a measure of the stiffness of the tissue. In Fig. 5(b) the force and indentation response of breast tissue in vivo can be expressed as $F=0.0526 e^{0.178 \delta}$, or $F=0.0526 e^{7.12 \lambda}$ with an initial thickness $40 \mathrm{~mm}$ at the measured position. The static expression of Eq.(1) can be extended to the dynamic case through the correspondence principle[1], so that Young's modulus is determined as $E=2\left(1-v^{2}\right) \kappa F / \delta l$. If we assume the breast tissue is incompressible, $E(\delta)=0.0526 e^{0.178 \delta}$. (The unit of Young's modulus is $\mathrm{MPa}$ ).

\section{Discussion and Conclusion}

In this study, a hand-held ultrasound indentation system that can acquire forcedisplacement response in vivo was presented. Non-invasive measures of biomechani- 
cal properties of soft tissue derived using this system were described. One novelty of our approach in comparison to others $[5,10]$ is we can quantitatively estimate not only integrated biomechanical properties of soft tissues (i.e. treating soft tissue as a homogeneous material) but also those of their internal components.

We presented phantom and in-vivo results. We showed that a linear elastic model could be used to model the phantom material over normal strain levels (up to $20 \%$ ). The Young's modulus estimated from experimental data via a mathematical solution, showed good agreement with 2D FEA and 3D FEA results, which showed that a plane-strain approximation was valid in this study. Considering the effect of different Poisson's ratios, friction factors, geometry sizes and boundary conditions, more detailed 2D and 3D FE analysis has been doing to define the applicable range of this mathematical solution. In vivo testing on breast tissue showed the viscoelasticity of the tissue. A pseudo-elastic model was fit to in vivo indentation data. In the indentation experiment, since the full deformation-time record and force-time record were acquired simultaneously, this meant we could investigate the hysteresis, relaxation and creep of the tissue in vivo. Future work will focus on investigating these features of soft tissues such as breast tissue.

In this study, the indentation rates ranged from $1.0 \mathrm{~mm} / \mathrm{s}$ to $10 \mathrm{~mm} / \mathrm{s}$, which was appropriate for manual control of the hand-held probe. It was shown that the indentation rate was insensitive to gelatin phantom measures. The same results were reported by Krouskop et al [22] for in-vitro tissues and Zheng et al [4] for in vivo plantar foot tissue.

Based on non-invasive ultrasound technique, the internal deformation field of soft tissue can be obtained. Consequently it is possible to evaluate abnormal and normal components of tissues. Both the Young's modulus and the Poisson's ratio can be reconstructed from this algorithm. In a primary study, using a finite element-based inverse reconstruction algorithm, the Young's moduli of a three-layer phantom were calculated. Good comparison was found between the reconstruction algorithms and experiments. This inverse reconstruct algorithm will be applied to clinical data in the future.

In this study, B-mode images were used to measure the displacement field. If raw RF signal data can be accessed from the ultrasound machine, this system can also act as a free-hand elastographic imaging system [23]. Because the force can be measured, quantitative Young's modulus can be determined rather than relative Young's modulus.

\section{References}

1. Fung, Y.C., Biomechanics-Mechanical Properties of Living Tissues. Second Edition, Springer-Verlag (1993)

2. Yamada, H., Strength of Biological Materials, Robert E. Krieger Publishing Company Huntington, New York.

3. Wang, B.C., Wang, G. R., Yan, D. H., Liu, Y. P. An Experimental Study on Biomechanical Properties of Hepatic Tissue Using A New Measuring Method. BioMedical Materials and Engineering. 2 (1992) 133-138

4. Zheng, Y.P.; Choi, Y.K.C.; Wong, K.; Chan, S.; Mak, A.F.T. Biomechanical Assessment of Plantar Foot Tissue in Diabetic Patients Using An Ultrasound Indentation System, Ultrasound in Medi.\&Biol, 26 (2000) 451-456 
5. Zheng, Y.P., Mak, A.F.T. An Ultrasound Indentation System for Biomechanical Properties Assessment of Soft Tissues in-vivo, IEEE Trans. Biomed. Engng. 43 (1996) 912-918

6. Mak, A.F.T., Liu, G.H.W. and Lee, S.Y. Biomechanical Assessment to Below-knee Residual Limb Tissue. J. Rehabil. Res. Dev. 31 (1994) 188-198

7. Miller, K, Chinzei, K., Orssengo G. and Bednarz P. Mechanical Properties of Brain Tissue In-Vivo. Experiment and Computer Simulation, J. Biomechanics, 33 (2000) 1369-1376

8. Hayes,W.C., Keer,L.M., Herrman,G and Mockros,L.F. A Mathematical Analysis for Indentation Tests of Articular Cartilage. J Biomechanics, 5 (1972) 541-551

9. Mak,A.F., Lai,W.M., Mow,V.C. Biphasic Indentation of Articular Cartilage-I. Theoretical Analysis. J. Biomechanics, 20 (1987) 703-714

10. Lyyra T., Jurvelin J., Pitkanem,P., Vaatainen U. and Kiviranta, Indentation Instrument for the Measurement of Cartilage Stiffness under Arthroscopic Control, Med. Eng. Phys. 17 (1995) 395-399

11. Kallel F, Bertrand M. Tissue Elasticity Reconstruction Using Linear Perturbation Method. IEEE Trans Med Imag, 15 (1996) 299-313.

12. Doyley M.M., Meaney P.M. and Bamber J.C., Evaluation of An Iterative Reconstruction Method for Quantitative Elastography, Phys. Med. Biol. 45 (2000) 1521-1540

13. Moulton M.J., Lawrence L.C., Ricardo L.A. An Inverse Approach Determining Myocardial Material Properties, J. Biomechanics 28 (1995) 935-948

14. Han L., J.A. Noble, M. Burcher, The Elastic Reconstruction of Soft Tissues, IEEE International Symposium on Biomedical Imaging: Macro to Nano (2002)

15. 15 Burcher, M., Han, L. and Noble J.A. Deformation Correction in Ultrasound Imaging Using Contact Force Information, Proc. IEEE Workshop on Mathematical Methods in Biomedical Image Analysis (2001)

16. Prager, R.W., Gee A.H., Berman L.. Stradx: Real-Time Acquisition and Visualisation of Freehand 3D Ultrasound, Cambridge University Engineering Dept. Technical Report, 1998.

17. Alblas, J.R, and Kuipers, M. Contact Problems of A Rectangular Block on An Elastic Layer of Finite Thickness, Part I :The Thin Layer, Acta,Mechica, 8 (1969) 133-145

18. Alblas, J.R, and Kuipers,M. Part II :The Thick Layer, Acta, Mechica ,9(1970) 1-12

19. Zhang, M., Zheng, Y.P and Mak, A.F.T. Estimating the Effective Young's modulus of Soft Tissue from Indentation Tests $\bullet$ Nonlinear Finite Element Analysis of Effects of Friction and Large Deformation, Med. Eng. Phys. 19, (1997) 512-517

20. Hayton, P.M., Brady, M., Smith, S. M. and Moore, N. A Non-rigid Registration Algorithm for Dynamic Breast MR Images, Artificial Intelligence, 114 (1999) 125-156.

21. IMSL Math/Library Manual, Visual Numerics, Inc.1997

22. Krouskop, T.A., Wheeler, T.M. Kallel, F. Garra, B.S. and Hall, T. Elastic Moduli of Breast and Prostate Tissues Under Compression. Ultrasonic Imaging 20,260-274 (1998)

23. Moyley, M.M., Bamber, J.C., Fuechsel, F. and Bush, B.L. A Freehand Elastographic Imaging Approach for Clinical Breast Imaging: System Development and Performance Evaluation, Ultrasound in Med.\& Biol. 27,1347-1357 (2001). 